\title{
COMITÊ EDITORIAL
}

EDITORA-CHEFE

Profa. Dra. Maria Claudia Bonadio

GESTORA FINANCEIRA

Profa. Dra. Maria de Fátima da Silva Costa Garcia de Mattos

ASSISTENTE EDITORIAL SÊNIOR

Profa. Ms. Gabriela Soares Cabral

ASSISTENTE EDITORIAL

Ana Luiza Monteiro

\section{CONSELHO CIENTÍFICO}

Profa. Dra. Agnes Roccamora (London College of Fashion, Inglaterra); Profa. Dra. Alessandra Vaccari (Università Iuav di Venezia, Itália); Profa. Dra. Alison Matthews David (Ryerson University, Canadá); Profa. Dra. Ana Claudia de Oliveira (Pontifícia Universidade Católica de São Paulo, Brasil); Profa. Dra. Ana Paula Cavalcanti Simioni (Instituto de Estudos Brasileiros, Universidade de São Paulo, Brasil); Profa. Dra. Caroline Evans (Central Saint Martins, Inglaterra); Profa. Dra. Denise Berruezo Portinari (Pontifícia Universidade Católica do Rio de Janeiro, Brasil); Prof. Dr. Fausto Viana (Escola de Comunicações e Arte, Universidade de São Paulo, Brasil); Prof. Dr. Frederic Godart (Insead, The Business School for the World, França); Profa. Dra. Giulia Ceriani (Università di Bergamo, Itália); Profa. Dra. Ivana Guilherme Simili (Universidade Estadual de Maringá, Brasil); Profa. Dra. Kathia Castilho (pesquisadora independente); Profa. Dra. Maria do Carmo Rainho (Arquivo Nacional, Brasil); Profa. Dra. Maria Eunice Souza Maciel (Universidade Federal do Rio Grande do Sul, Brasil); Profa. Dra. Paula Maria Guerra Tavares (Universidade do Porto, Portugal); Prof. Dr. Paulo Keller (Universidade Federal do Maranhão, Brasil); Profa. Dra. Regina Root (William \& Mary, Estados Unidos); Profa. Dra. Renata Pitombo (Universidade Federal do Recôncavo da Bahia, Brasil); Profa. Dra. Rita Morais de Andrade (Universidade Federal de Goiás, Brasil); Prof. Dr. Ronaldo de Oliveira Corrêa (Universidade Federal do Paraná, Brasil); Profa. Dra. Sofia Pantouvaki (Aalto University, Finlândia); Profa. Dra. Vânia Carneiro Carvalho (Museu Paulista, Universidade de São Paulo, Brasil).

NOMINATA DOS PARECERISTAS DO ANO DE 2019 (EDIÇÕES 25, 26 E 27):

Adair Marques Filho (Universidade Federal de Goiás); Aliana Aires (Universidade Federal do Piauí); Aline Monçores (Universidade Veiga de Almeida); Ana Helena Barbosa Bezerra de Souza (Universidade Federal de Minas Gerais); Ana Lúcia de Castro (Universidade Estadual Paulista); Angela Lucia Silva Figueiredo (Universidade Federal do Recôncavo da Bahia); Angélica Adverse (Universidade do Estado de Minas Gerais); Angélica Vier Munhoz (Universidade do Vale do Taquari); Anne Anicet Ruthschilling (Contextura); Arlei Damo (Universidade Federal do Rio Grande do Sul); Carlos Costa (Universidade Federal de São Carlos); Carmen Lucia Soares (Universidade Estadual de Campinas); Carolina Bassi de Moura (Universidade Federal do Estado do Rio de Janeiro); Caroline Soares (Universidade Federal de Santa Catarina); Clemara Bidarra (Fundação Armando Álvares Penteado); Cristiano Mezzaroba (Universidade Federal de Sergipe); Dalmir Rogerio Pereira (Universidade Federal de Goiás); Daniela Novelli (Universidade do Estado de Santa Catarina); Débora El Jaick Andrade (Universidade Federal Fluminense); Denise Castilhos de Araujo (Universidade do Vale do Rio dos Sinos); Edinéia Pereira da Silva Betta (Centro Universitário de Brusque); Edivaldo Góis (Universidade Estadual de Campinas); Edmilson Felipe da Silva (Pontifícia Universidade Católica de São Paulo); Elisabeth Murilho (Universidade Federal de Juiz de Fora); Elizete Messias (Universidade Federal de Alagoas); Emanuele Biollo Magnus (Universidade Feevale); Fabiana Jordão Martinez (Universidade Federal de Goiás); Fausto Viana (Universidade de São Paulo); Flávia Cera (Pontifícia Universidade Católica do Paraná); Flavio Sabra (Instituto Federal do Rio de Janeiro); Francione Oliveira Carvalho (Universidade Federal de Juiz de Fora); Gabriela Ordones Penna (Centro Universitário Una); Glaucia Villas Bôas (Universidade Federal do Rio de Janeiro); Heloísa Pontes (Universidade Estadual de Campinas); Irina Aragão (Pontifícia Universidade Católica do Rio de Janeiro); Isabel Cristina Italiano (Universidade de São Paulo); Joana Carolina Schossler (Universidade Estadual de Campinas); 
Joana Monteleone (Universidade Federal do ABC); João Dalla Rosa Júnior (Serviço Nacional de Aprendizagem Industrial - Centro de Tecnologia da Indústria Química e Têxtil); Juliana de Mello Moraes (Universidade Regional de Blumenau); Juliana Schmitt (Universidade Federal de Juiz de Fora); Leda Costa (Universidade Federal Fluminense); Luiza Aguiar dos Anjos (Universidade Federal de Minas Gerais); Luz Neira Garcia (pesquisadora independente); Madson de Oliveira (Universidade Federal do Rio de Janeiro); Maíra Zimmermann de Andrade (Fundação Armando Álvares Penteado); Manuela do Corral Vieira (Universidade Federal do Pará); Marco Aurélio Paz Tella (Universidade Federal da Paraíba); Margarida Maria Adamatti (Universidade Federal de São Carlos); Maria Cristina Volpi Nacif (Universidade Federal do Rio de Janeiro); Maria de Fatima da Silva Costa Garcia de Mattos (Centro Universitário Moura Lacerda); Maria do Carmo Rainho (Arquivo Nacional); Maria Eduarda Araújo Guimarães (Centro Universitário do Serviço Nacional de Aprendizagem Comercial de São Paulo); Maria Izabel Branco Ribeiro (Fundação Armando Álvares Penteado); Mariana Bassi Sutter (University of Leeds); Mariane Pisani (Universidade Federal do Tocantins); Marinês Ribeiro (Universidade Tecnológica Federal do Paraná); Maristela Abadia Fernandes Novaes (Universidade Federal de Goiás); Marizilda dos Santos Menezes (Universidade Estadual Paulista); Marko Monteiro (Universidade Estadual de Campinas); Miqueli Michetti (Universidade Federal da Paraíba); Mylene Mizrahi (Universidade Federal Rural do Rio de Janeiro); Olga Pepece (Universidade Estadual de Maringá); Patrícia Pavesi (Universidade Federal do Espírito Santo); Paulo Fernandes Keller (Universidade Federal do Maranhão); Priscila Vieira (Universidade Federal do Paraná); Raphael Bispo (Universidade Federal de Juiz de Fora); Raphael Castanheira Scholl (Universidade Feevale); Renata Cardoso da Silva (Universidade Federal da Bahia); Rita de Morais Andrade (Universidade Federal de Goiás); Rochelle Cristina dos Santos (Universidade Federal de Santa Catarina); Ronaldo de Oliveira Corrêa (Universidade Federal do Paraná); Samila Batistoni (Universidade de São Paulo); Sílvia Maria Carbone (Universidade Brás Cubas); Solange Mezabarba (Instituto Elpídio Donizetti); Taisa Sena Vieira (Pontifícia Universidade Católica de Minas Gerais); Tarcisio D’Almeida (Universidade Federal de Minas Gerais); Valdenise Leziér Martyniuk (Pontifícia Universidade Católica de São Paulo); Valquíria Aparecida dos S. Ribeiro (Universidade Tecnológica Federal do Paraná); Victor Melo (Universidade Federal do Rio de Janeiro); Viviane Teixeira (Universidade Federal de Pelotas); Wagner Xavier de Camargo (Universidade Federal de São Carlos); Wladimir Machado (Universidade Federal do Vale do São Francisco).

CAPA

Marcelo Max a partir da ilustração Celebrate, de Andrés Leonardo Caballero Piza

CRÉDITO DAS ILUSTRAÇÕES DA CAPA E DO MIOLO DA REVISTA

Andrés Leonardo Caballero Piza

PROJETO GRÁFICO | DIREÇÃO DE ARTE

Marcelo Max

REVISÃO DOS TEXTOS EM PORTUGUÊS

Márcia Moura e Ana Carolina Carvalho

REVISÃO DOS ABSTRACTS

Larissa Andrioli

CONTATO

dobras@abepem.com.br

SITE

https://dobras.emnuvens.com.br

REVISTA DOBRAS e-ISSN 2358-0003 | ISSN impresso 1982-0313 v. 1 n. 1 da Dobras/2007.

ASSOCIAÇÃO BRASILEIRA DE ESTUDOS E PESQUISAS EM MODA (ABEPEM)

Rua Cardoso de Almeida, 788, cj. 144 - CEP: 05013-001. São Paulo - SP. 